\title{
Clinical characteristics of Kawasaki disease and concurrent pathogens during isolation in COVID-19 pandemic
}

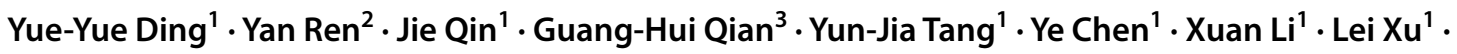 \\ Chun-Hong Qiao ${ }^{4} \cdot$ Ling Sun ${ }^{1} \cdot$ Hai-Tao Lv ${ }^{1}$ (1)
}

Received: 8 February 2021 / Accepted: 8 April 2021 / Published online: 23 June 2021

(c) Children's Hospital, Zhejiang University School of Medicine 2021

\begin{abstract}
Background The aim of this study is to explore the characteristics of Kawasaki disease (KD) and concurrent pathogens due to a stay-at-home isolation policy during coronavirus disease 2019 (COVID-19) epidemic.

Methods All patients with KD admitted between February and April in 2015-2020, were classified into before (group 1, in 2015-2019) and after (group 2, in 2020) isolation groups. A total of 4742 patients [with $\mathrm{KD}(n=98)$ and non-KD $(n=4644)$ ] referred to Mycoplasma pneumoniae (MP) and virus detection were analyzed in 2020. Clinical characteristics, laboratory data, and 13 pathogens were analyzed retrospectively.

Results Group 2 had a significantly increased incidence of $\mathrm{KD}(0.11 \%)$ with 107 patients compared to that of group $1(0.03 \%)$ with 493 patients. The comparisons of oral mucosal change, strawberry tongue, desquamation of the fingertips, cervical lymphadenopathy and neutrophil percentage decreased in group 2 compared to group 1 . The infection rate of MP increased significantly in group $2(34.7 \%)$ compared to group $1(19.3 \%)$, while the positive rate of viruses decreased significantly in group 2 (5.3\%) compared to group 1 (14.3\%). In 2020, the positive rate of MP infection increased significantly in patients with KD compared to the increase in patients with non-KD. The infection rate of MP for younger children aged less than 3 years old was higher in group 2 than in group 1.

Conclusion Compared with the characteristics of KD from 2015 to 2019 years, the incidence of KD was increased in 2020 and was accompanied by a high incidence of MP infection, especially in younger children (less than 3 years old) during the isolation due to COVID-19 pandemic.
\end{abstract}

Keywords Kawasaki disease $\cdot$ Concurrent pathogens $\cdot$ Mycoplasma pneumoniae $\cdot$ COVID-19 $\cdot$ Isolation

Hai-Tao Lv

lvhaitao@suda.edu.cn

1 Cardiology Department, Children's Hospital of Soochow University, Suzhou 215025, China

2 Department of Radiology, Huashan Hospital of Fudan University, 12 Mid Wulumuqi Road, Shanghai 200040, China

3 Institute of Pediatric Research, Children's Hospital of Soochow University, Suzhou 215025, China

4 Department of Biostatistics and Computational Biology, School of Life Science, Fudan University, Shanghai 200433, China

\section{Introduction}

The current pandemic of coronavirus disease 2019 (COVID-19) caused by severe acute respiratory syndrome coronavirus 2 (SARS-CoV-2) has resulted in more than 98,669,492 cases and 2,112,037 deaths worldwide. The incidence of COVID-19 in children aged $<18$ years is lower than that in adults and accounts for $1-2 \%$ of all patients with COVID-19. In Suzhou city, eastern China, only one child was diagnosed with COVID-19, accounting for $1.15 \%$ of the total 87 patients diagnosed with COVID-19 positive during the pandemic. Data from China and the USA suggest that pediatric COVID19 may be less severe than that in adults [1, 2]. However, an outbreak of severe, Kawasaki-like disease at the Italian epicenter of COVID-19 has been reported [3]; therefore, we have focused on the characteristics of 
Kawasaki disease (KD) in children during the COVID19 pandemic period.

$\mathrm{KD}$ is a self-limited acute vasculitis and is the most common cause of acquired heart disease in children in developing countries. The etiology and risk factors of KD are still unknown despite the fact that half a century has passed since the first case series was reported in Japan [4]. A viral origin (likely RNA) of the disease is the most accepted hypothesis [5-8]. The diagnosis of KD is mainly based on clinical characteristics and laboratory data according to some criteria established by the Japanese Kawasaki Disease Research Committee in 2005 [9] and updated by the American Heart Association in 2017 [10]. Currently, the generally accepted pathogenetic hypothesis supports an inflammatory cascade triggered by one or more unidentified pathogens in genetically predisposed subjects $[3,11]$. Previous studies have revealed that seasonal variations in the onset of KD may be related to exposure to environmental factors including unspecified infectious pathogens [12]. However, common infectious pathogens also have synchronized regulation of seasonal variability similar to KD, such as Mycoplasma pneumoniae (MP) [13, 14], influenza viruses [15] and rotaviruses [16]. To date, the pathogenesis and clinical manifestations of KD are still inadequately understood, especially in the case of out-of-home viral transmission control.

Prior studies have suggested that KD is closely associated with viral $[17,18]$ and bacterial involvement $[19,20]$ and even with normal flora that originate from the host or that ubiquitously exist in human reservoirs, which are influenced by environmental changes [21]. During the global spread of the SARS-CoV-2 pandemic, children's exposure to infection sources was greatly reduced due to the stay-at-home isolation policy in China. It is unknown whether the stay-at-home isolation could have affected the morbidity of KD and what the clinical characteristics of KD are.

The aim of the present study was to describe the incidence and clinical features of KD in the patients admitted to our hospital during the stay-at-home isolation. Comparisons of the incidence and features of KD were performed using data from the past 5 years, to help pediatricians understand the pathogenetic changes and clinical features of KD in children with less exposure to external infection sources.

\section{Methods}

\section{Clinical data collection}

We respectively reviewed the medical records of patients diagnosed with KD and admitted to our unit and along with records of other contemporaneous pediatric patients with non-KD from February 1 to April 30 in 2015-2020. All KD patients were categorized into group 1 (from 2015 to 2019) and group 2 (from 2020 under the isolation policy after the outbreak of COVID-19). SARS-CoV-2 polymerase chain reaction (PCR) tests were performed and the patients with negative results could be admitted to our hospital in 2020 . In addition, a total of 4742 patients, who underwent MP and virus detection from February 1 to April 30 in 2020, were classified into groups of $\mathrm{KD}(n=98)$ and non-KD $(n=4644)$ for statistical analysis. This retrospective study was approved by the Ethics Committee of the Children's Hospital of Soochow University with a waiver for written informed consent by the participants' legal guardian or next of kin.

We collected data on epidemiology, clinical characteristics, laboratory tests and echocardiography including age, gender, KD-associated manifestations, treatment, and clinical outcomes. Laboratory tests were performed on the first day of hospitalization and included the following acute phase reactants: white blood cell count (WBC), percentage of neutrophils, hemoglobin, platelet count, serum albumin, C-reactive protein (CRP), erythrocyte sedimentation rate (ESR), alanine aminotransferase (ALT), aspartate aminotransferase (AST) and lactate dehydrogenase (LDH). In addition, the results of infectious pathogen test were also collected, including MP antibody (MP-IgG, MP-IgM) or MP-DNA by PCR, Chlamydia pneumoniae (CP), EpsteinBarr virus (EBV), cytomegalovirus (CMV), coxsackievirus, respiratory viruses consisting of adenovirus (ADV), respiratory syncytial virus (RSV), influenza virus A \& B (Inf A, B), parainfluenza virus $1,2 \& 3$ (Pinf 1, 2, 3), rotavirus and SARS-CoV-2. Clinical features consisting of response to intravenous immunoglobulin (IVIG), intravenous steroid injection regimen, presence of coronary artery lesions (CALs) or no (nCALs) also were reviewed.

\section{Definition of Kawasaki disease}

Complete KD (cKD) was diagnosed in accordance with the criteria established by the Japanese Kawasaki Disease Research Committee before 2017 and with the guidelines on the diagnosis and management of KD by the American Heart Association after 2017 [10]. The criteria consisted of the presence of fever for at least 5 days together with at least 4 of the 5 following principal clinical features: (1) erythema and cracking of lips, strawberry tongue, and/or erythema of oral and pharyngeal mucosa; (2) bilateral bulbar conjunctival congestion without exudate; (3) rash: maculopapular, diffuse erythroderma, or erythema multiforme-like; (4) erythema and edema of the hands and feet in the acute phase and/or periungual desquamation in the subacute phase; and (5) cervical lymphadenopathy ( $\geq 1.5 \mathrm{~cm}$ diameter) and usually unilateral. In the presence of at least four principal clinical features, particularly when redness and swelling of the hands and feet were present, the diagnosis of KD can be made with 4 days of fever. 
Incomplete $\mathrm{KD}$ (also referred to as atypical $\mathrm{KD}, \mathrm{iKD}$ ) should be considered in any infant or child with prolonged unexplained fever, fewer than four of the principal clinical findings, and compatible laboratory or echocardiographic findings. Non-response to IVIG was defined as persistent or recrudescent fever $\geq 36$ hours after the initial IVIG infusion.

CALs were defined by the standard $Z$ value $\geq 2.0$ of the internal lumen diameter of the coronary artery, based on echocardiographic evaluation performed before IVIG infusion and within 2 weeks of illness onset or iKD when CALs were confirmed. Coronary artery aneurysm was defined as a segmental internal diameter of any segment $\geq 1.5$ times greater than that of an adjacent segment. Giant coronary aneurysm was defined as a segmental internal diameter $\geq 8 \mathrm{~mm}$. The steroid regimen was an intravenous pulse of methylprednisolone at a dose of $30 \mathrm{mg} / \mathrm{kg}$ for $2-3$ hours, administered once daily for 1-3 days. The same sonographer measured all of the indicators, and the same pediatric cardiologist reviewed all echocardiograms.

\section{Mycoplasma pneumoniae detection and evaluation}

Nasopharyngeal secretions were obtained within 24 hours after admission. The pharyngeal swab was placed in storage solution and then was shaken thoroughly. Following centrifugation at 12,000 r/minute for 5 minutes, the sediment was removed. Then, $50 \mu \mathrm{L}$ of nucleic acid extract was added to the precipitation solution after discarding the supernatant, and the sample was placed in a water bath at $100{ }^{\circ} \mathrm{C}$ for 10 minutes, and then was centrifuged at $12,000 \mathrm{r} / \mathrm{min}$ ute for 10 minutes. Fluorescent quantitative real-time PCR (BIO-RAD iCycler, USA) was performed for MP DNA, and samples with a $\mathrm{Ct}$ value $>30$ copies $/ \mathrm{mL}$ and an $\mathrm{S}$-shaped amplification curve were considered positive. An MP-negative sample was defined as having an amplification curve that was not $\mathrm{S}$-shaped or a $C_{\mathrm{t}}$ value $\leq 30$.

Venous blood samples $(2 \mathrm{~mL})$ were collected, and the serum was isolated for serological analysis. IgM antibodies were measured using the chemiluminescence method by an iFlash MP IgM kit and an iFlash MP IgG kit (Yahuilong Biotechnology Company, Shenzhen, China). The results were expressed in relative luminous intensity. A cut-off intensity $(\mathrm{COI})<0.9 \mathrm{AU} / \mathrm{mL}$ was considered antibody IgM negative, and a $\mathrm{COI}<0.9-1.1 \mathrm{AU} / \mathrm{mL}$ was considered antibody $\operatorname{IgM}$ weakly positive, and a $\mathrm{COI} \geq 1.1 \mathrm{AU} / \mathrm{mL}$ was considered antibody IgM strongly positive. The detection process for IgG antibodies was similar to that for IgM antibodies using relative luminous intensity: $\mathrm{COI}<24 \mathrm{AU} / \mathrm{mL}$ was negative for $\mathrm{IgG}, 24-36 \mathrm{AU} / \mathrm{mL}$ was weakly positive and $\geq 36 \mathrm{AU} / \mathrm{mL}$ was strongly positive for IgG. All operations were carried out in strict accordance with the manufacturer's instructions.
The diagnosis of MP infection was based on serology and PCR findings. Both the presence of IgM antibodies and positive PCR results were used as sufficient criteria for current MP infection.

\section{Virus detection and evaluation}

\section{Epstein-Barr virus and cytomegalovirus detection}

Venous blood $(2 \mathrm{~mL})$ from all children was collected and placed in an EDTA-K2 anti-coagulant or pro-coagulant tube. Mononuclear cells were separated from anti-coagulant blood with lymphocyte separation solution, and blood serum was directly separated from pro-coagulant blood by centrifugation, which was stored in the refrigerator at $-20{ }^{\circ} \mathrm{C}$ for later use. EBV-DNA and CMV-DNA detection was performed in strict accordance with the reagent instructions. A LightCycler 480 fluorescence quantitative PCR instrument (Roche Applied Science Inc., Switzerland) was used, and the kit was provided by Hunan Shengxiang Biotechnology Co., China. DNA determination and results were as follows: for those with a viral load of 100 copies $/ \mathrm{mL}$ or above, the value was taken directly; for those with a viral load between 10 and 100 copies $/ \mathrm{mL}$, the test was repeated once and the average value was taken. Those with a viral load $\geq 10$ copies $/ \mathrm{mL}$ were judged to be positive, and a viral load $<10$ copies $/ \mathrm{mL}$ were judged to be negative.

\section{Respiratory virus detection}

Nasopharyngeal secretions were obtained by an aseptic suction tube inserted through the nose $7-8 \mathrm{~cm}$ into the pharynx and then absorbed by negative pressure. The exfoliated cell smear was dried naturally and then fixed with cold acetone. The corresponding monoclonal antibody $(10 \mu \mathrm{L})$ was added, the sample was incubated and then stained for 30 minutes in the dark. After rinsing with phosphate buffer saline, the sample was observed under a fluorescence microscope. Nasopharyngeal washes were evaluated using the Vircell Respiratory Virus Panel (Vircell, S.L.Parque Tecnológico de la Salud, Granada, Spain), which detects CP, ADV, RSV, Inf A, Inf B, Pinf 1, Pinf 2, and Pinf 3 in a total of eight respiratory viruses and subtypes.

\section{Rotavirus detection}

Fecal samples (100 mg per sample) were collected from children 24 hours after admission. After collection, the samples were immediately placed into sterile extraction tubes for preservation. Group A rotaviruses were detected using rotavirus diagnostic kits (Zhuhai Lizhu Reagent Co., Ltd., China) and the colloidal gold method was selected for rotavirus detection. 


\section{SARS-CoV-2 detection}

Nasopharyngeal swabs or oropharyngeal swabs were obtained within 24 hours before admission. The pharyngeal swab was placed in storage solution, and then thoroughly shaken. 2019-nCoV nucleic acid kit (Zhongshan Da An Gene Co. Ltd., China) was used. Nucleic acid was extracted by DA3200 nucleic acid extraction instrument (Daan Gene Co. Ltd., Zhongshan, China), and product amplification by ABI7500 PCR instrument (Thermo Fisher Scientific, American). Select open reading frame $1 \mathrm{ab}($ ORF $1 a b)$ and nucleoprotein $(N)$ gene region designed primers and probes. Target 1 (ORF $1 a b)$, forward primer: 5'-CCCTGTGGGTTTTAC ACTTAA-3', reverse primer: 5'-ACGATTGTGCATCAG CTGA-3', probe: 5'-FAM-CCGTCTGCGGTATGTGGA AAGGTTATGG-BHQ1-3'. Target $2(N)$, forward primer: 5'-GGGGAACTTCTCCTGCTAGAAT-3', reverse primer: 5'-CAGACATTTTGCTCTCAAGCTG-3', probe: 5'-FAMTTGCTGCTGCTTGACAGATT-TAMRA-3'. Samples gene of $O R F 1 a b$ and $N$ with a $C_{\mathrm{t}}$ value $\leq 40$ copies $/ \mathrm{mL}$ and an $\mathrm{S}$-shaped amplification curve were considered positive.

\section{Statistical analysis}

The characteristics of the study participants were summarized using descriptive statistics: number and percentage for categorical variables; mean and standard deviations for continuous variables. Differences in categorical variables were assessed with the $\chi^{2}$ test and Fisher's exact test $(n<5)$. The $t$ test was used to determine if the means of two groups of data differed. The Spearman's rank correlation is introduced to analyze the correlation between variables. A $P$ value less than 0.05 was considered statistically significant. All statistical analyses were performed using $\mathrm{R}$ language and environment ( $\mathrm{R}$ version 3.6.1).

\section{Results}

A total of 645 patients with KD were reviewed in this study between February 1 and April 30 from 2015 to 2020. Forty-five patients were excluded due to incomplete data. The remaining 600 patients were included in the study. The age at onset ranged from 1 to 159 months with a median of 29.1 months. The male to female ratio was $1.34: 1$. There were 493 cases in group 1 from 22,749 inpatients between February 1 and April 30 in 2015-2019 and 107 cases in group 2 from 3782 inpatients in the same period in 2020 (Fig. 1a). There were 493 cases in group 1 from 1,671,168 outpatients between February 1 and April 30 in 2015-2019 and 107 cases in group 2 from 93,454 outpatients in the same period in 2020 . The incidence of KD showed a significant increase in group 2 with $13.27 \%$ for inpatients and $0.11 \%$ for outpatients compared with group 1 with $7.76 \%$ for inpatients and $0.03 \%$ for outpatients, respectively (all $P<0.001$ ) (Fig. 1b). The incidence rate of KD between February 1 and April 30 in 2015-2020 is described in Fig. 1c and the peak of KD onset is in 2020.

As shown in Table 1, the comparisons of clinical features between groups 1 and 2 demonstrated that the incidence of oral mucosal change, strawberry tongue, desquamation of the fingertips, and cervical lymphadenopathy were significantly decreased in group 2 compared with group 1 (all $P<0.001)$. Other factors including gender, sex, conjunctival congestion, rash, edema of the extremities, desquamation of the perineal region, change in Bacillus Calmette Guerin vaccine site, IVIG response, cKD or iKD, and intravenous steroid injection regimen were not significantly different between the two groups (Table 1).

As shown in Table 1, comparisons of laboratory findings demonstrated that only the value of neutrophils\% was significantly increased in group 2 compared with group $1(P=0.003)$. No statistically significant differences in
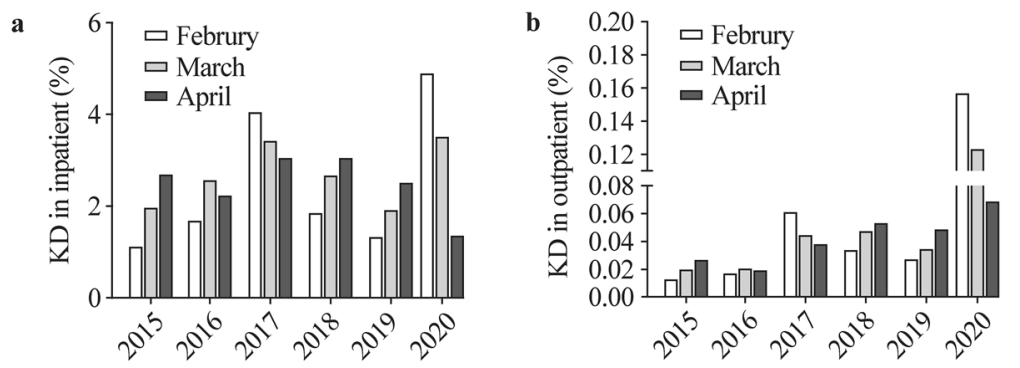

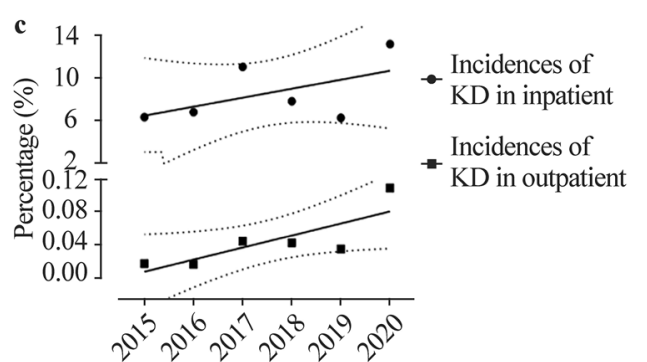

Fig. 1 The incidences of Kawasaki disease (KD) for inpatients and outpatients between February 1st to April 30th from 2015 to 2020. a The histogram shows the incidences of KD for inpatients between February 1st to April 30th from 2015 to 2020; b the histogram shows the incidences of KD for outpatients between February 1st and April 30th from 2015 to 2020; $\mathbf{c}$ the incidence of KD for inpatients and outpatients in each year are shown in percentage with $95 \%$ confidence interval 
Table 1 Clinical and laboratory features of Kawasaki disease under the pandemic of COVID-19 or not in recent 6 years

\begin{tabular}{|c|c|c|c|}
\hline Variables & Group $1(n=493)$ & Group $2(n=107)$ & $P$ \\
\hline Male, $n(\%)$ & $281(57.0)$ & $63(58.9)$ & 0.80 \\
\hline Age (mon), median (range) & $21(1-159)$ & $22(2-111)$ & 0.50 \\
\hline$<6, n(\%)$ & $43(8.7)$ & $8(7.5)$ & 0.80 \\
\hline $6-12, n(\%)$ & $66(13.4)$ & $12(11.2)$ & 0.70 \\
\hline $12-36, n(\%)$ & $251(50.9)$ & $56(52.3)$ & 0.90 \\
\hline $36-60, n(\%)$ & $73(14.8)$ & $21(19.3)$ & 0.27 \\
\hline$\geq 60, n(\%)$ & $60(12.2)$ & $10(9.4)$ & 0.51 \\
\hline Conjunctival congestion, $n(\%)$ & $426(86.4)$ & $88(82.2)$ & 0.29 \\
\hline Oral mucosal changes, $n(\%)$ & $426(86.4)$ & $77(72.0)$ & $<0.001$ \\
\hline Strawberry tongue, $n(\%)$ & $328(66.5)$ & $45(42.1)$ & $<0.001$ \\
\hline Rash, $n(\%)$ & $353(71.6)$ & $85(80.2)$ & 0.07 \\
\hline Edema of extremities, $n(\%)$ & $208(42.2)$ & $45(42.1)$ & 1.00 \\
\hline Desquamation of the fingertips, $n(\%)$ & $156(31.6)$ & $13(12.2)$ & $<0.001$ \\
\hline Desquamation of the perineal region, $n(\%)$ & $120(24.3)$ & $19(17.8)$ & 0.17 \\
\hline Cervical lymphadenopathy, $n(\%)$ & $445(90.3)$ & $79(73.8)$ & $<0.001$ \\
\hline Changes at BCG site, $n(\%)$ & $86(17.4)$ & $16(15.0)$ & 0.57 \\
\hline IVIG responders, $n(\%)$ & $381(77.3)$ & $84(78.5)$ & 0.90 \\
\hline Complete KD, $n(\%)$ & $407(82.6)$ & $91(85.1)$ & 0.67 \\
\hline Steroid regimen intravenous injection, $n(\%)$ & $38(7.7)$ & $7(6.5)$ & 0.84 \\
\hline CAL or not CAL, $n(\%)$ & $83(17.4)$ & $19(17.8)$ & 0.90 \\
\hline KD shock syndrome, $n(\%)$ & $0(0)$ & $0(0)$ & NA \\
\hline Macrophage activation syndrome (KD-MAS), $n(\%)$ & $3(0.6)$ & $2(1.9)$ & 0.10 \\
\hline White cell count $\left(\times 10^{9} / \mathrm{L}\right)$, mean $\pm \mathrm{SD}$ & $15.3 \pm 6.1$ & $14.6 \pm 5.4$ & 0.30 \\
\hline Neutrophils $\%$, mean \pm SD & $59.9 \pm 21.9$ & $65.3 \pm 15.5$ & 0.003 \\
\hline Hemoglobin $(\mathrm{g} / \mathrm{L})$, mean \pm SD & $108.7 \pm 12.7$ & $110.2 \pm 11.5$ & 0.30 \\
\hline Platelet count $\left(\times 10^{9} / \mathrm{L}\right)$, mean $\pm \mathrm{SD}$ & $375.1 \pm 136.4$ & $363.4 \pm 133.3$ & 0.40 \\
\hline Serum albumin $(\mathrm{g} / \mathrm{L})$, mean $\pm \mathrm{SD}$ & $39.0 \pm 14.4$ & $40.6 \pm 3.6$ & 0.30 \\
\hline $\mathrm{CRP}(\mathrm{mg} / \mathrm{L})$, mean $\pm \mathrm{SD}$ & $81.1 \pm 59.4$ & $74.8 \pm 49.8$ & 0.30 \\
\hline $\operatorname{ESR}(\mathrm{mm} / \mathrm{h})$, mean $\pm \mathrm{SD}$ & $38.0 \pm 24.7$ & $39.3 \pm 22.3$ & 0.60 \\
\hline $\operatorname{ALT}(\mathrm{U} / \mathrm{L})$, mean $\pm \mathrm{SD}$ & $50.4 \pm 72.1$ & $60.8 \pm 77.1$ & 0.20 \\
\hline $\mathrm{AST}(\mathrm{U} / \mathrm{L})$, mean $\pm \mathrm{SD}$ & $54.8 \pm 81.1$ & $61.2 \pm 92.7$ & 0.50 \\
\hline $\mathrm{LDH}(\mathrm{mmol} / \mathrm{L})$, mean $\pm \mathrm{SD}$ & $401.7 \pm 145.4$ & $400.8 \pm 148.2$ & 1.00 \\
\hline
\end{tabular}

COVID-19 coronavirus disease 2019, KD Kawasaki disease, IVIG intravenous immunoglobulin, $C A L$ coronary artery lesions, CRP C-reactive protein, $E S R$ erythrocyte sedimentation rate, $A L T$ alanine aminotransferase, $A S T$ aspartate aminotransferase, $L D H$ lactate dehydrogenase, $S D$ standard deviation, $N A$ not available. Bold letters indicating significance

hemoglobin, serum albumin, WBC, CRP, ESR, ALT, AST and LDH were observed between the two groups.

The incidence rate of positive MP increased significantly in group $2(34.7 \%, 34 / 98)$ compared to group $1(19.3 \%$, $81 / 420)(P=0.0015)$. However, the incidence rate of positive viruses was significantly decreased in group $2(5.3 \%$, $4 / 76)$ compared to group $1(14.3 \%, 61 / 428)(P=0.04)$. To rule out the possible effect of the number of referrals to the laboratory, we calculated the detection rates of MP and special viruses in KD. The percentage of MP detected in KD was not significantly different between group 1 (85.2\%, $420 / 493)$ and group $2(91.6 \%, 98 / 107)(P=0.112)$. The percentage of viruses detected significantly decreased in group
$2(71.0 \%, 76 / 107)$ compared to group $1(86.6 \%, 428 / 493)$ (Fig. 2a). The specific information of the virus infection in group 1 and group 2 was shown by Fig. 2 b.

A marked increase in the incidence of MP infection was found in KD patients $(34.7 \%, 34 / 98)$ compared with non-KD patients $(16.8 \%, 778 / 4644)$ during the stay-at-home isolation policy from February 1 to April 30 in 2020. However, no significant difference in the incidence rate of other virus infections was found between KD patients $(5.3 \%, 4 / 98)$ and non-KD patients $(5.9 \%, 274 / 4644)$ (Table 2). In addition, children of MP positive non-KD group were significantly older (median age $=51$ months) than children of MP positive KD group (median age $=$ with 22 months). 

Total patients in group 1 $(n=493)$

\section{Total patients in group 2}

$$
(n=107)
$$

\section{MP detection patients $420(85.2)$}

Virus detection patients 428 (86.6)
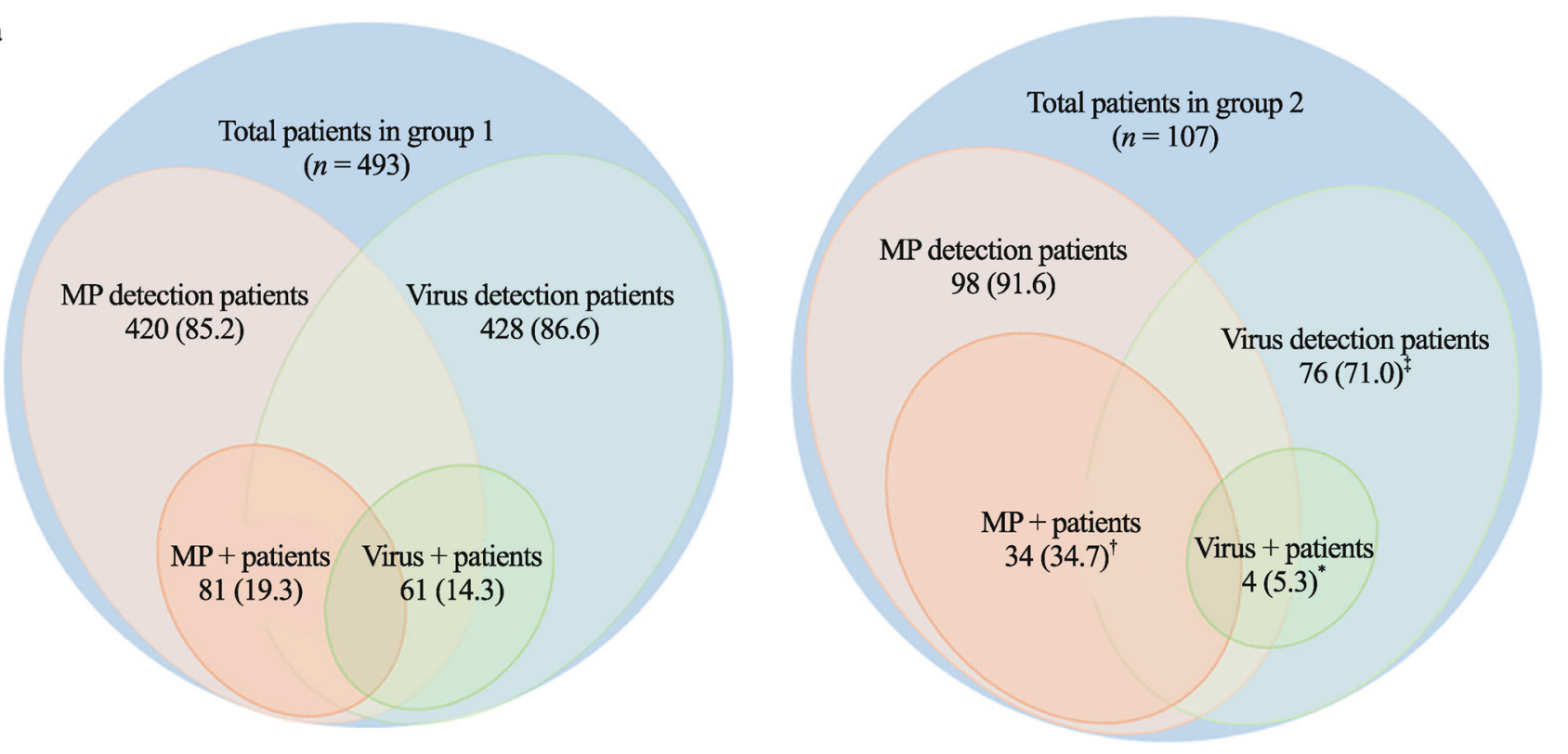

b

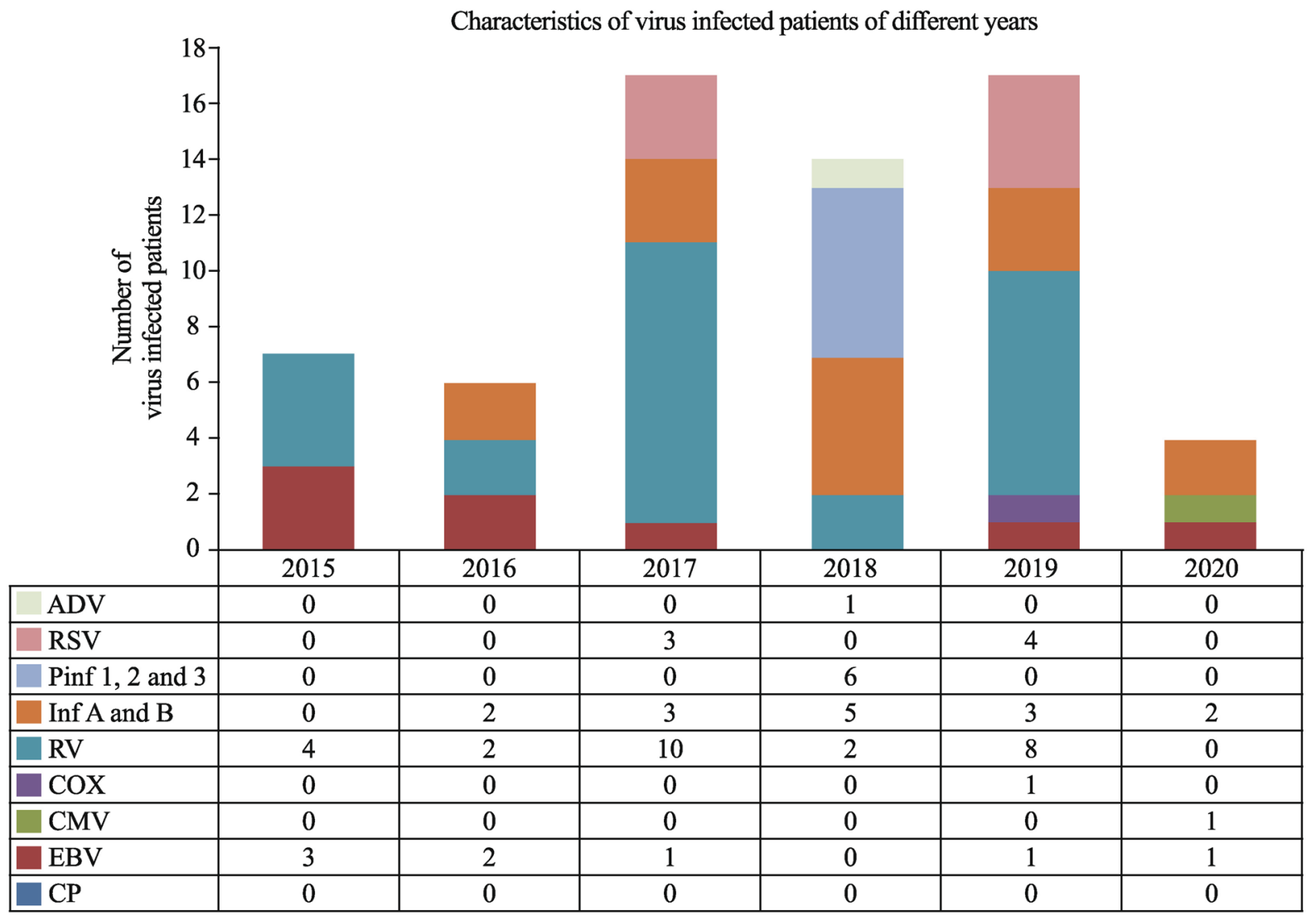

Fig. 2 Characteristics of virus- and MP-infected patients for the two groups in the years of 2015-2020. a The detection and positive ratios of virus- and MP-infection of KD patients for groups of 1 and 2 from 2015 to 2020 are demonstrated by Wenn graphs. Values are $n(\%) ; *, \dagger$ and $\ddagger$ indicate $P<0.05, P<0.01$ and $P<0.001$, respectively; $\mathbf{b}$ bar graph shows the detailed num- ber of virus-infected patients in each year from 2015 to 2020, MP Mycoplasma pneumoniae, $N$ neutrophils, $C P$ chlamydia pneumoniae, $E B V$ Epstein-Barr virus, $C M V$ cytomegalovirus, $C O X$ coxsackievirus, $R V$ rotavirus, Inf $A, B$ influenza virus A \& B, Pinf 1, 2, 3 parainfluenza virus $1,2 \& 3, R S V$ respiratory syncytial virus, $A D V$ adenovirus 
Table 2 Etiological detection in Kawasaki disease and outpatient among February to April in 2020

\begin{tabular}{lllll}
\hline Variables & Non-KD group $(n=4644)$ & KD group $(n=107)$ & $P$ & OR (95\% CI) \\
\hline Male, $n(\%)$ & $2674(57.6)$ & $63(58.9)$ & 0.80 & NA \\
Age (mon), median (range) & $51(1-216)$ & $22(2-111)$ & $<\mathbf{0 . 0 0 1}$ & NA \\
MP+, $n(\%)$ & $778(16.8)$ & $34(34.7)$ & $<\mathbf{0 . 0 0 1}$ & $2.6(1.73-4.03)$ \\
Virus+, $n(\%)$ & $274(5.9)$ & $4(5.3)$ & 1.00 & $0.9(0.32-2.44)$ \\
\hline
\end{tabular}

KD Kawasaki disease, MP Mycoplasma pneumonia. OR odds ratio, $C I$ confidence interval, $N A$ not available. Bold letters indicating significance

We conducted a statistical analysis of MP positive rates in different age groups of $\mathrm{KD}$ and found that the ratios of MP positive in 2020 were statistically higher for the children less than 3 years old compared with those in the same period from 2015 to 2019. The ratios of MP positive for the children less than 12 months old were $23.8 \%$ (5/21) for the year of 2020 and $13.1 \%$ (14/107) for the past 5 years, respectively. The ratios of MP positive for the children from 12 to 36 months old were $17.6 \%$ (36/204) for the year of 2020 and $37.3 \%$ (19/51) for the past 5 years, respectively. With the increase of age, the MP positive rate increased, but the difference for the children of more than 3 years old between two groups was not statistically significant (Table 3).

\section{Discussion}

In this study, the incidence of KD during the 3-month period of isolation in 2020 was markedly increased compared to the average incidence of the same period during 2015-2019 in Suzhou, east China. The higher incidence of KD in 2020 was accompanied by the increased MP infection in the younger children aging less than 3 years old. Under the strict stayat-home isolation policy, person-to-person interaction was extensively reduced due to the COVID-19 pandemic [22]. The greatly reduced environmental exposure to infectious agents could theoretically have played a role in limiting the $\mathrm{KD}$ onset according to the available evidence, which indicates that the pathogenesis of KD is closely associated with various viruses or microbes [23]. Therefore, to explore the pathogenesis of $\mathrm{KD}$, in the case of almost no transmission of infection, we carefully analyzed the clinical and laboratory features, including the results of various infectious pathogens, such as MP, respiratory virus and rotavirus, by comparing the incidences of these features between years of 2020 and pre-2020 (2015-2019) and between groups of KD and non-KD in the year of 2020.

There was a higher incidence of MP infection among the KD patients from February 1st to April 30th in 2020 than in those of the same period from 2015 to 2019. This result was confirmed by ruling out the effect of MP detection rate between group 1 and group 2. According to Tang et al.'s study [24], the incidence of KD associated with the increased incidence of MP infection in the older children. MP infection tended to occur in older populations with KD (median age $=25$ months); however, we found that MP infection had a closer association with an increasing KD in the younger children of less than 36 months, especially for those infants aged less than 12 months. The exact mechanism still remains unknown, but it could be attributed to dysregulation of innate immune response and to the immaturity of the adaptive immunity of a host according to a prior assumption by Kano et al. [25].

Significantly, MP infection had a closer positive association with KD, especially among younger children (less than 12 months of age). At present, although we cannot find the causative pathogen of $\mathrm{KD}$, but younger infants are thought to be more susceptible to KD once they are infected by MP.

Table 3 The positive of Mycoplasma pneumonia detection in Kawasaki disease of various age groups from 2015 to 2020

\begin{tabular}{|c|c|c|c|c|c|}
\hline \multirow[t]{2}{*}{ Age } & \multicolumn{2}{|c|}{ Group 1 (2015-2019) } & \multicolumn{2}{|l|}{ Group 2 (2020) } & \multirow[t]{2}{*}{$P$} \\
\hline & Positive number & Detection number & Positive number & Detection number & \\
\hline Total number, $n(\%)$ & $81(19.3)$ & $420(100)$ & $34(34.7)$ & $98(100)$ & NA \\
\hline$<12$ mon, $n(\%)$ & $14(13.1)$ & $107(25.5)$ & $5(23.8)$ & $21(21.4)$ & $<0.001$ \\
\hline $12-36$ mon, $n(\%)$ & $36(17.6)$ & $204(48.6)$ & $19(37.3)$ & $51(52.0)$ & 0.01 \\
\hline $36-60$ mon, $n(\%)$ & $16(27.6)$ & $58(13.8)$ & $6(35.4)$ & $17(17.3)$ & 0.38 \\
\hline$>60$ mon, $n(\%)$ & $15(29.4)$ & $51(12.1)$ & $4(44.4)$ & $9(9.2)$ & 0.56 \\
\hline
\end{tabular}

NA not available. Bold letters indicating significance 
To date, a huge amount of effort is devoted to find the pathogenic factors and mechanism of KD, but with little effect. A major reason is the inability to find proper subjects for the research among a naturally social environment. However, the strict isolation due to COVID-19 pandemic provides us with an unprecedented chance in which the transmission of various pathogenic agents is virtually blocked. Under this condition, the results may be much closer to the real causal pathogenesis of $\mathrm{KD}$, although they may depart from traditional ideas. Current understanding on the pathogenesis of $\mathrm{KD}$ is a cascade triggered by one or more infectious agents and is affected not only by genetic factors but also by seasonality and ethnicity [26]. Several confounding factors have hindered the identification of the specific causative agent of KD. However, the present study successfully dodged the disturbance of seasonality and person-to-person transmission to a great extent, which may explain why our results differed from those in previous studies. The present study provides new knowledge to understand the pathogenesis of KD.

Furthermore, the positive rate of concurrent viruses in $\mathrm{KD}$ was significantly decreased. From February 1 to April 30 of the COVID-19 pandemic in this year, Suzhou city implemented a strict stay-at-home isolation policy, and accordingly local citizens have greatly reduced their interaction and stayed home. Therefore, this has greatly reduced exposure to virus-associated transmission due to isolation and has made it possible to observe changes in other concurrent pathogens in KD. But why did the MP infectious rate significantly increase when virus infectious rate deceased? One reason may be that infants got along with adult caregivers too long and lacked of outdoor activity during the stay-at-home isolation.

With regard to clinical findings, a significantly decreased incidence of changes in the extremities (desquamation of the fingertips), oral mucosal changes, strawberry tongue and cervical lymphadenopathy in KD were observed during the 3-month study period in 2020 during the COVID-19 pandemic, which was lower than the previous average rate, and could be related to the decreased incidence of viral pathogens and/or increased MP incidence. We consider that one or more pathogens could trigger the pathogenesis of KD. Although, in view of the current understanding, virus-associated specific clinical features are confounded in non-virus-associated patients with $\mathrm{KD}[3,27,28]$, a reduction of above clinical features could be suggestive of non-virus-associated KD. Whether these clinical findings help to identify virus-associated KD requires further validation. Other clinical features, including age at onset, male to female ratio, conjunctival congestion, rash, edema of the extremities, desquamation of perineal region, changes in Bacillus Calmette Guerin site, IVIG response, cKD or iKD, intravenous steroid injection regimen, CALs or nCALs, were not indicative for identifying the features during the stay-at-home isolation of the COVID-19 pandemic. More significantly, despite the decreased incidence of virus infection in group 2 compared with group 1, the same frequency of IVIG response and CALs or nCALs was observed, which could imply a loose association between IVIG resistance, CALs and concurrent virus infection in patients with KD [27]. Prior studies also indicated that coronary artery abnormality (and the response to IVIG) in the children with KD may be related to the extent of inflammation (such as greater CRP), not to whatever pathogen is present [29-31]. In addition, during laboratory testing, the increased value of neutrophil percentage differing from that of the past 5 years, could be associated with the higher ratio of concurrent MP infection.

During the COVID-19 pandemic, our observational study was carried out under the strict stay-at-home isolation policy, including wearing masks and maintaining social distancing. When a child needed to go to the children's hospital, he underwent rigorous epidemiological investigation, and SARS-CoV-2 PCR detections were performed before admission to the unit between February and April in 2020. If a positive patient was found by PCR or by antibody test, the patient would be immediately isolated to designate infectious disease hospital instead of staying in the children's hospital. So the inpatients in this unit were all negative of SARS-CoV-2 PCR detection.

There are several other limitations in this study. We noted that the incidence of KD in April 2020 was significantly lower than that in February and March 2020, which was completely different from the previous increase in morbidity during April compared with February and March in each year from 2015 to 2019. Therefore, the observation period should be extended to increase our knowledge on the incidence of KD in subsequent months. In addition, this was a single-center study, sample selection bias could have existed in the analysis of Suzhou city due to statistical omission in a small population of child care centers. $Z$ scores for CAL diagnosis were not used prior to 2017.

To sum up, during the isolation period, these findings are meaningful in guiding the clinical practice. The incidence of KD was increased and was accompanied by increased MP infection in the children less than 3 years old under the situation that the environmental exposure was restrained.

Author contributions DYY, RY and QJ contributed equally to this study as first co-authors. DYY and RY contributed to conceptualization, data curation, methodology, formal analysis, investigation and writing. QJ, QGH, TYJ, CY, LX, XL, and SL contributed to data curation, investigation and project administration. QCH contributed to methodology and formal analysis. LHT contributed to conceptualization, investigation, funding acquisition and supervision. All authors read and approved the final manuscript. 
Funding: This work was supported by the grants from the National Natural Science Foundation of China (Nos. 81870365, 81971477 and 81970436), the National Youth Foundation of China (No. 81800437), the Jiangsu Provincial Medical Young Talents (QNRC2016756 and QNRC2016764), the Shanghai Science and Technology Support Project for Medicine (No. 18411967300).

\section{Compliance with ethical standards}

Ethical approval The study was approved by the Ethics Committee of the Children's Hospital of Soochow University (reference number 2020CS066) with a waiver for written informed consent by the participants' legal guardian/next of kin.

Conflict of interest Authors declare that they have no conflict of interest.

Data availability The datasets generated during and/or analyzed during the current study are available from the corresponding author on reasonable request.

\section{References}

1. CDC COVID-19 Response Team. Coronavirus Disease 2019 in Children-United States, February 12-April 2, 2020. MMWR Morb Mortal Wkly Rep. 2019;2020:422-6.

2. Dong YY, Mo X, Hu YB, Qi X, Jiang F, Jiang ZY, et al. Epidemiology of COVID-19 among children in China. Pediatrics. 2020;145:e20200702.

3. Verdoni L, Mazza A, Gervasoni A, Martelli L, Ruggeri M, Ciuffreda M, et al. An outbreak of severe Kawasaki-like disease at the Italian epicentre of the SARS-CoV-2 epidemic: an observational cohort study. Lancet. 2020;395:1771-8.

4. Kawasaki T. Acute febrile mucocutaneous syndrome with lymphoid involvement with specific desquamation of the fingers and toes in children. Arerugi. 1967;16:178-222 (in Japanese).

5. Nagao Y, Urabe C, Nakamura H, Hatano N. Predicting the characteristics of the aetiological agent for Kawasaki disease from other paediatric infectious diseases in Japan-ERRATUM. Epidemiol Infect. 2016;144:493.

6. Rowley AH, Baker SC, Orenstein JM, Shulman ST. Searching for the cause of Kawasaki disease-cytoplasmic inclusion bodies provide new insight. Nat Rev Microbiol. 2008;6:394-401.

7. Rowley AH, Baker SC, Shulman ST, Fox LM, Takahashi K, Garcia FL, et al. Cytoplasmic inclusion bodies are detected by synthetic antibody in ciliated bronchial epithelium during acute Kawasaki disease. J Infect Dis. 2005;192:1757-66.

8. Rowley AH, Baker SC, Shulman ST, Garcia FL, Guzman-Cottrill JA, Chou P, et al. Detection of antigen in bronchial epithelium and macrophages in acute Kawasaki disease by use of synthetic antibody. J Infect Dis. 2004;190:856-65.

9. Ayusawa M, Sonobe T, Uemura S, Ogawa S, Nakamura Y, Kiyosawa N, et al. Revision of diagnostic guidelines for Kawasaki disease (the 5th revised edition). Pediatr Int. 2005;47:232-4.

10. McCrindle BW, Rowley AH, Newburger JW, Burns JC, Bolger $\mathrm{AF}$, Gewitz M, et al. Diagnosis, treatment, and long-term management of Kawasaki disease: a scientific statement for health professionals from the American Heart Association. Circulation. 2017;135:e927-99.

11. Franco A, Shimizu C, Tremoulet AH, Burns JC. Memory T-cells and characterization of peripheral T-cell clones in acute Kawasaki disease. Autoimmunity. 2010;43:317-24.

12. Uehara R, Belay ED. Epidemiology of Kawasaki disease in Asia, Europe, and the United States. J Epidemiol. 2012;22:79-85.
13. Lan YL, Li SX, Yang DH, Zhou JF, Wang YS, Wang JH, et al. Clinical characteristics of Kawasaki disease complicated with Mycoplasma pneumoniae pneumonia: a retrospective study. Medicine (Baltimore). 2020;99:e19987.

14. Vitale EA, La Torre F, Calcagno G, Infricciori G, Fede C, Conti $\mathrm{G}$, et al. Mycoplasma pneumoniae: a possible trigger of kawasaki disease or a mere coincidental association? Report of the first four Italian cases. Minerva Pediatr. 2010;62:605-7.

15. Dunning J, Thwaites RS, Openshaw PJM. Seasonal and pandemic influenza: 100 years of progress, still much to learn. Mucosal Immunol. 2020;13:566-73.

16. Crawford SE, Ramani S, Tate JE, Parashar UD, Svensson L, Hagbom M, et al. Rotavirus infection. Nat Rev Dis Primers. 2017;3:17083.

17. Quiat D, Kula T, Shimizu C, Kanegaye JT, Tremoulet AH, Pitkowsky Z, et al. High-throughput screening of Kawasaki disease sera for antiviral antibodies. J Infect Dis. 2020;222:1853-7.

18. Huang SH, Chen CY, Weng KP, Chien KJ, Hung YM, Hsieh $\mathrm{KS}$, et al. Adenovirus infection and subsequent risk of Kawasaki disease: a population-based cohort study. J Chin Med Assoc. 2020;83:302-6.

19. Leung C. Clinical characteristics of COVID-19 in children: are they similar to those of SARS? Pediatr Pulmonol. 2020;55:1592-7.

20. Matsubara K, Fukaya T, Miwa K, Shibayama N, Nigami H, Harigaya $\mathrm{H}$, et al. Development of serum IgM antibodies against superantigens of Staphylococcus aureus and Streptococcus pyogenes in Kawasaki disease. Clin Exp Immunol. 2006;143:427-34.

21. Rhim JW, Kang HM, Han JW, Lee KY. A presumed etiology of Kawasaki disease based on epidemiological comparison with infectious or immune-mediated diseases. Front Pediatr. 2019;7:202.

22. Bassareo PP, Melis MR, Marras S, Calcaterra G. Learning from the past in the COVID-19 era: rediscovery of quarantine, previous pandemics, origin of hospitals and national healthcare systems, and ethics in medicine. Postgrad Med J. 2020;96:633-8.

23. Principi N, Rigante D, Esposito S. The role of infection in Kawasaki syndrome. J Infect. 2013;67:1-10.

24. Tang YJ, Yan WH, Sun L, Huang J, Qian WG, Hou M, et al. Kawasaki disease associated with Mycoplasma pneumoniae. Ital J Pediatr. 2016;42:83.

25. Kano Y, Mitsuyama Y, Hirahara K, Shiohara T. Mycoplasma pneumoniae infection-induced erythema nodosum, anaphylactoid purpura, and acute urticaria in 3 people in a single family. J Am Acad Dermatol. 2007;57(Suppl 2):S33-5.

26. Nakamura A, Ikeda K, Hamaoka K. Aetiological significance of infectious stimuli in Kawasaki disease. Front Pediatr. 2019;7:244.

27. Turnier JL, Anderson MS, Heizer HR, Jone PN, Glodé MP, Dominguez SR. Concurrent respiratory viruses and Kawasaki disease. Pediatrics. 2015;136:e609-14.

28. Narita M. Classification of extrapulmonary manifestations due to Mycoplasma pneumoniae infection on the basis of possible pathogenesis. Front Microbiol. 2016;7:23.

29. Zheng XL, Wu WC, Zhang Y, Wu G. Changes in and significance of platelet function and parameters in Kawasaki disease. Sci Rep. 2019;9:17641.

30. Downie ML, Manlhiot C, Latino GA, Collins TH, Chahal N, Yeung RS, et al. Variability in response to intravenous immunoglobulin in the treatment of Kawasaki disease. J Pediatr. 2016;179:124-30.

31. Rigante D, Valentini P, Rizzo D, Leo A, De Rosa G, Onesimo R, et al. Responsiveness to intravenous immunoglobulins and occurrence of coronary artery abnormalities in a single-center cohort of Italian patients with Kawasaki syndrome. Rheumatol Int. 2010;30:841-6.

Publisher's Note Springer Nature remains neutral with regard to jurisdictional claims in published maps and institutional affiliations. 